\title{
NUEVO REGISTRO DE DISTRIBUCIÓN DEL PUERCO ESPÍN DEL NORTE ERETHIZON DORSATUM EN CHIHUAHUA, MÉXICO
}

Gatica-Colima, A., Navarrete-Laborde, B., Ortiz-González, A. \& Rosas-Rosas, O. C. 2014. New distributional record of the North American porcupine Erethizon dorsatum in Chihuahua, Mexico. Acta Zoológica Mexicana (n. s.), 30(2): 399-402.

ABSTRACT. We document the occurrence of a North American porcupine Erethizon dorsatum in the Sierra Juárez, municipality of Juárez, Chihuahua, Mexico, some $100 \mathrm{~km}$ east of the nearest record, this represents the seventh record for the state.

El puerco espín del norte Erethizon dorsatum (también nombrado recientemente $E$. dorsata) está ampliamente distribuido en Canadá y Estados Unidos, y en algunas localidades del norte de México (Jones \& Genoways 1968). Se ha reportado a Erethizon dorsatum en el estado de Chihuahua en seis localidades (Fig. 1): Ojo Palomos Viejo y a $8 \mathrm{~km}$ al SE de Colonia Juárez (Jones \& Genoways 1968); Rancho El Uno, Los Bejucos, Los Novillos y Rancho Ojitos (List et al. 1999).

Entre abril de 2003 y abril de 2004 se realizó un inventario de los mamíferos en la Sierra Juárez, en el municipio de Juárez Chihuahua. Con apoyo de cinco trampas cámaras y la identificación de excretas y huellas, se registraron seis especies de mediana talla (Ortiz 2004) y tres de mayor talla (Navarrete 2004).

El 3 de noviembre de 2003 (ca 08:30 h) durante un recorrido en la porción Oeste de la sierra (31 $39^{\prime} 39.96^{\prime}$ 'N , 106 $34^{\circ} 12.33^{\prime}$ 'W a 1,427 msnm), se logró tomar una fotografía del puerco espín con una cámara análoga Nikon N60, lente de 28-80 mm. La fotografía (Fig. 2) está depositada en la Colección Científica de Vertebrados de la Universidad Autónoma de Ciudad Juárez (Photo Voucher CCV-UACJ-32-03). Este es el séptimo registro documentado de la especie para el estado de Chihuahua, ca 100 kilómetros (62 millas) al Este del registro más cercano conocido como Ojo Palomos Viejo. 


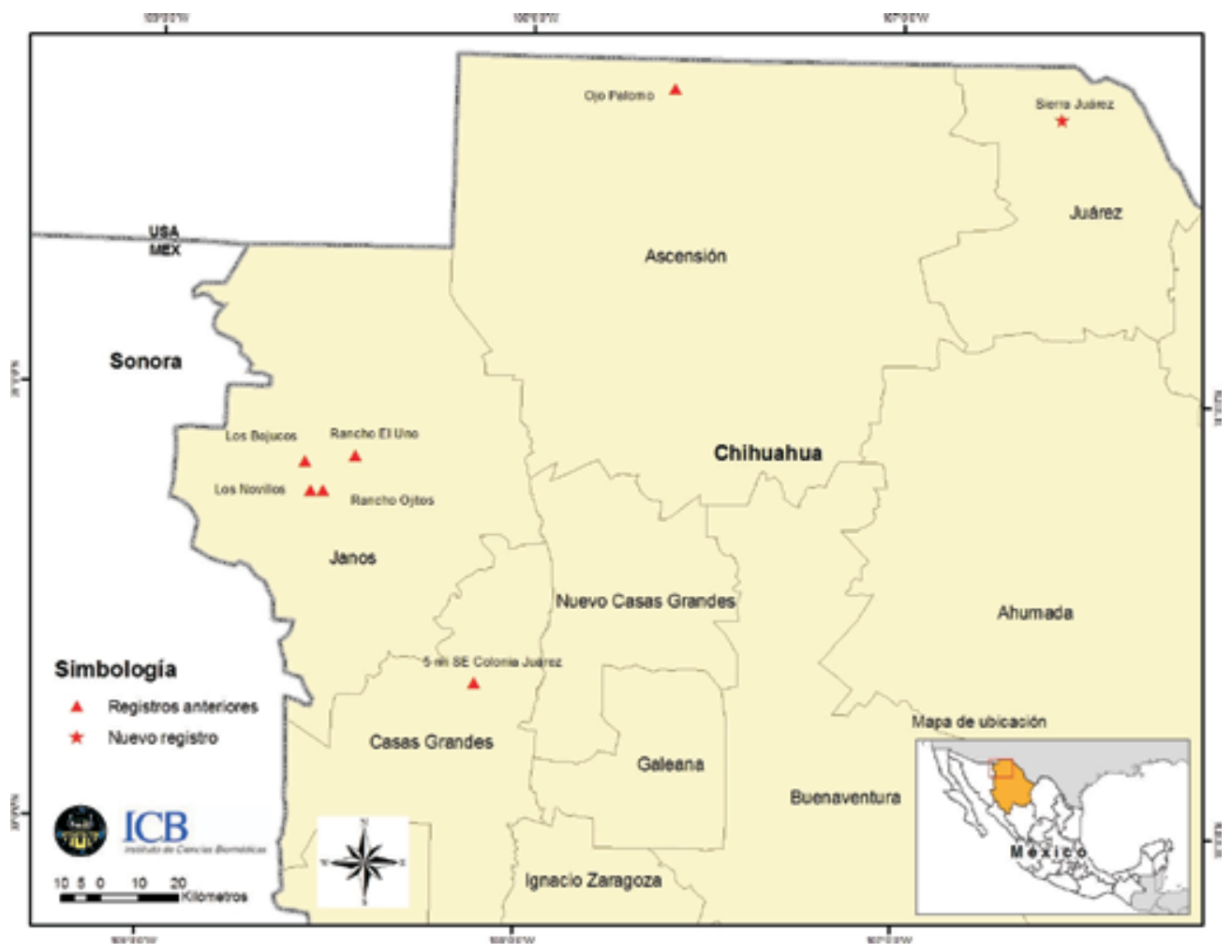

Figura 1. Registros del puerco espín norteño Erethizon dorsatum en Chihuahua (Elaboró Nora Reyes)

Se conoce que las madrigueras preinvernales del puerco espín se ubican cerca de las áreas favoritas de alimentación que consisten en troncos huecos (Olson \& Lewis 1999). La vegetación donde se ubicó el puerco espín fue matorral desértico, dominado por la lechuguilla Agave lechugilla y Yucca sp., lo que es consistente con la literatura, ya que se ha reportado en el matorral desértico de Arizona (Reynolds 1957).

De acuerdo con Anderson (1972) la ocurrencia de puerco espín es esporádica en Sonora, Oeste de Texas, Sur de Arizona y Nuevo México, al igual que el norte de Chihuahua. Se ha reportado que la especie ha estado expandiendo su distribución en las últimas décadas, a lo largo de terrenos relativamente abiertos en gran parte del Noroeste de Texas (Baird et al. 2009).

Se ha documentado que la baja densidad del puerco espín aunado a la caza indiscriminada son las causas por las cuales la especie está amenazada en México (Leopold 1965; Ceballos \& Navarro 1991). La población más numerosa de puerco espín reportada para México se encuentra en Janos, Chihuahua (List et al. 1999). Actual- 


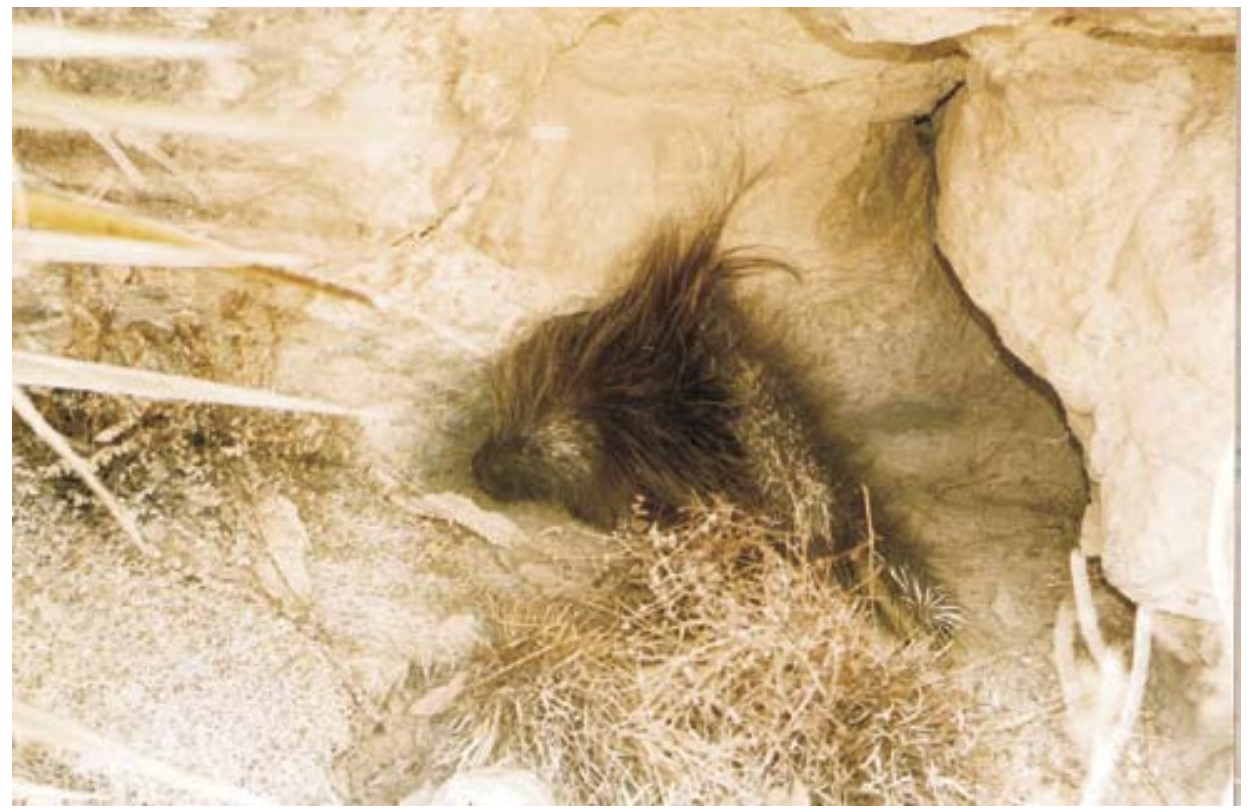

Figura 2. Puerco espín norteño Erethizon dorsatum de la Sierra Juárez, Juárez, Chihuahua (Foto por Armine Ortiz-González).

mente el puerco espín del norte E. dorsatum está en la categoría de Peligro de Extinción (P) dentro de la NOM-059-SEMARNAT-2010 (DOF, 2010); sin embargo, poco se conoce de la biología y ecología de esta especie en México.

Recientemente, la sierra Juárez ha sido transformada por la construcción de una carretera (Camino Real) en la porción Este pero el impacto por la fragmentación del hábitat sobre la vida silvestre es desconocido. Con este registro en la Sierra Juárez, se incrementa considerablemente el área de distribución de la especie Erethizon dorsatun. El conocimiento de nuevas localidades de puerco espín es importante en la conservación de la especie.

\section{LITERATURA CITADA}

Anderson, S. 1972. Mammals of Chihuahua. Taxonomy and distribution. Bulletin of the American Museum of Natural History, 148: 149-410.

Baird, A. B., Pauly, G. B., Hall, D. W. \& Travis, L. J. 2009. Records of the porcupine (Erethizon dorsatum) from the eastern margins of the Edwards Plateau of Texas. Texas Journal of Science, 61: 65-67.

Ceballos, G. \& Navarro, D. 1991. Diversity and conservation of the Mexican mammals, pp. 167-198. In: M. A. Mares and D. J. Schmidly (Eds.). Topics in Latin American Mammalogy: History, Biodiversity and Conservation. University of Oklahoma Press, Norman, Oklahoma, EUA. 
DOF. 2010. Norma Oficial Mexicana NOM-059-SEMARNAT-2010, Protección ambiental-Especies nativas de México de flora y fauna silvestre-categorías de riesgo y especificaciones para su inclusión, exclusión o cambio-Lista de especies en riesgo. Diario Oficial de la Federación.

Jones, K. \& Genoways, H. 1968. Distribution of the Porcupine Erethizon dorsatum in Mexico. Mammalia, 32: 709-712.

Leopold, A. S. 1965. Fauna Silvestre de México. Aves y Mamíferos de Caza. Instituto Mexicano de Recursos Naturales Renovables. PAX-México. México, D. F.

List, R., Ceballos, G. \& Pacheco, J. 1999. Status of the North American porcupine (Erehizon dorsatum) in Mexico. Southwestern Naturalist, 44: 400-404.

Navarrete, L. B. 2004. Mamíferos mayores de la Sierra Juárez, Chihuahua. Tesis de Licenciatura en Biología, Universidad Autónoma de Ciudad Juárez.

Olson, R. \& Lewis, A. M. 1999. Porcupine Ecology and Damage Management Techniques for Rural Homeowners. Cooperative Extension Service, School of Agriculture B-1073, University of Wyoming, Laramie, Wyoming.

Ortiz, G. A. 2004. Mamíferos de mediana talla de la Sierra Juárez, Chihuahua. Tesis de Licenciatura en Biología, Universidad Autónoma de Ciudad Juárez.

Reynolds, H. G. 1957. Porcupine behavior in the desert-shrub type of Arizona. Journal of Mammalogy, 38: 418-419.

\section{Ana GATICA-COLIMA, ${ }^{1 *}$ BenJamín NAVARRETE-LABORDE, ${ }^{1}$ ARMine ORTIZ-GONZÁLEZ ${ }^{1} \&$ OCTAVIO C. ROSAS-ROSAS ${ }^{2}$}

${ }^{1}$ Universidad Autónoma de Ciudad Juárez. Instituto de Ciencias Biomédicas. Programa de Biología. Laboratorio de Ecología y Biodiversidad Animal. Anillo Envolvente del PRONAF y Estocolmo s/n. Juárez, Chihuahua, México. CP 32310.

${ }^{2}$ Colegio de Posgraduados Campus San Luis. Agustín de Iturbide \#73. CP 78622, Salinas de Hidalgo, San Luis Potosí. México.

*Autor de Correspondencia: <agatica@uacj.mx> 